\title{
Enjoyment of life and declining physical function at older ages: a longitudinal cohort study
}

\author{
Andrew Steptoe DSc, Cesar de Oliveira PhD, Panayotes Demakakos PhD, Paola Zaninotto PhD
}

Competing interests: None declared.

This article has been peer reviewed.

\section{Correspondence to:}

Andrew Steptoe,

a.steptoe@ucl.ac.uk

CMAJ 2014. DOI:10.1503 /cmaj.131155

\begin{abstract}
Background: Positive affective well-being (i.e., feelings of happiness and enjoyment) has been associated with longer survival and reduced incidence of serious illness. Our objective was to discover whether enjoyment of life also predicted a reduced risk of functional impairment over an 8-year period in a large population sample.
\end{abstract}

Methods: We carried out a prospective analysis involving 3199 men and women aged 60 years or older from the English Longitudinal Study of Ageing. Enjoyment of life was assessed by questionnaire. Outcomes were impairment in 2 or more activities of daily living and changes in gait speed on a walking test. Covariates included sociodemographic factors, baseline health, depressive symptoms, impairment of mobility and health behaviours.
Results: Two or more impaired activities of daily living developed among $4.4 \%, 11.7 \%$ and $16.8 \%$ of participants in the high, medium and low enjoyment-of-life tertiles, respectively. After adjustment for covariates, the odds of impaired activities of daily living developing were 1.83 (95\% confidence interval 1.13-2.96) in the low compared with high tertile. Gait speed after 8 years was also related to baseline enjoyment of life after adjustment for gait speed and other covariates at baseline $(p<0.001)$. We obtained similar results when we limited analyses to participants younger than 70 years at baseline.

Interpretation: This is an observational study, so causal conclusions cannot be drawn. But our results provide evidence that reduced enjoyment of life may be related to the future disability and mobility of older people.
$\mathrm{T}$ here is accumulating evidence that greater subjective well-being is associated with longer survival and reduced incidence of coronary heart disease and stroke. ${ }^{1-4}$ Associations are particularly striking for positive affective well-being (i.e., feelings of happiness and enjoyment) and appear to be independent of comorbidities. ${ }^{5}$ Less is known about the relation of positive affective wellbeing to functional decline and the incidence of disability at older ages.$^{6-8}$ An appreciation of such associations may aid in clinical care and in understanding the processes of functional decline, which is important given that decline in physical function in turn predicts mortality. ${ }^{9}$ Here, we describe the relation between enjoyment of life and decline in physical function in a nationally representative population cohort of older adults. Functional decline was assessed with gait speed, and disability by the development of impaired activities of daily living. We considered sociodemographic, health and behavioural factors to estimate the independent effect of enjoyment of life on physical function outcomes.

\section{Methods}

The English Longitudinal Study of Ageing is a longitudinal panel study of men and women aged 50 or older, representative of people living in England. ${ }^{10}$ Data for wave 1 were collected in 2002/03, and follow-up took place every 2 years thereafter, with a nurse visit and biomarker assessment every 4 years (www.ifs.org.uk/ELSA). For our analysis, we used wave 1 as the baseline and assessed outcomes from wave 5 (2010/11). The 8-foot walk test was carried out only by participants aged 60 or older, so all our analyses were based on this age group. Complete data on enjoyment of life, all covariates and impaired activities of daily living were available from 3199 participants. Of these, 2965 completed the walk test in wave 1, and 2507 in waves 1 and 5 , so changes in gait speed were analyzed in this subgroup.

\section{Measures}

For our analysis, the main exposure was enjoyment of life, assessed with the pleasure subscale from the CASP-19 quality-of-life instrument (a 19-item scale covering control, autonomy, self-realization 
and pleasure $)^{11}$ as described previously. ${ }^{12}$ The 4 items of the subscale were "I enjoy the things that I do," "I enjoy being in the company of others," "On balance, I look back on my life with a sense of happiness" and "I feel full of energy these days"; each was rated on a 4-point scale from 0 (never) to 3 (often), and ratings were summed to generate a total score. The Cronbach $\alpha$ for the scale was 0.65 , and the test-retest correlation was 0.61 over 2 years and 0.53 over 8 years.

Impairments of activities of daily living were assessed during personal interviews using a measure developed for the Health and Retirement Study..$^{13}$ Respondents were asked if they had any difficulty with 6 activities because of a physical, mental, emotional or memory problem. Individual items are listed in Appendix 1, available at www.cmaj.ca/lookup/suppl/doi:10.1503 /cmaj.131155/-/DC1. Difficulties that were expected to last less than 6 months were excluded. We used 2 or more impaired activities of daily living to define impairment, because a single item may have fewer implications for social care. However, results of our analysis of the development of a single impaired activity of daily living were similar to those reported here. Gait speed was assessed with two 8-foot walking tests from a standing start; the tests were carried out in the participants' homes under the supervision of a trained interviewer. Individuals who had health conditions or disabilities that prevented walking were not eligible for the test.

Age was divided into 3 categories $(60-69,70$ 79 and $\geq 80 \mathrm{yr}$ ) for the purpose of analysis. Education was classified into lower (no qualifications), intermediate (high school qualifications) and higher (college and higher degrees). Socioeconomic status was estimated by total household wealth, including financial wealth (i.e., savings and investments), the value of any home and other property (less any mortgage), the value of any business assets and physical wealth (e.g., artwork and jewellery), and net of debt, ${ }^{10}$ and was divided into quintiles. Marital status was classified as married or not married, ethnicity as white European origin or nonwhite, and employment status as paid employment or not. General health was assessed by discovering whether participants had a limiting, long-standing illness. Respondents were also asked whether a doctor told them they had coronary heart disease, cancer, diabetes, stroke, chronic lung disease, arthritis or clinical depression. Depressive symptoms were measured using the 8item Centre for Epidemiologic Studies Depression Scale (CES-D),${ }^{14}$ and a score of 4 or greater was used to indicate marked symptomatology, as in previous studies. ${ }^{15}$ Mobility was assessed by asking about activities involving leg mobility and arm function (e.g., walking 100 yards, reaching or extending arms above shoulder level) and impairment was modelled as a binary variable. Current smoking, engagement in vigorous or moderate activity at least once per week, and consumption of alcohol daily or almost daily were also recorded.

\section{Statistical analysis}

Scores on the enjoyment-of-life measure were negatively skewed, averaging 10.3 (standard deviation [SD] 1.6) on the 12-point scale. Participants were divided into tertiles of enjoyment of life for the primary analyses; allowing for ties, this resulted in 669 in the high (score 12), 1792 in the medium (score 10 or 11) and 738 in the low (score $<10)$ tertile. We modelled the development of impaired activities of daily living using logistic regression, and we modelled gait speed using linear regression. Participants with 2 or more impaired activities of daily living at baseline were excluded from the longitudinal analysis of activities of daily living. Five sequentially adjusted models were tested in the prediction of impaired activities of daily living over the 8-year follow-up period. Model 1 included enjoyment of life, age, sex and baseline impaired activities of daily living (i.e., 0 or 1). Baseline demographic factors (i.e., wealth, education, ethnicity, marital status and employment status) were added in model 2. Health indicators (i.e., limiting, longstanding illness; coronary heart disease; cancer; stroke; chronic lung disease; diabetes; and arthritis) were added in model 3. We included depression (raised CES-D score and recent depressive illness) in model 4 to test whether associations between enjoyment of life and impaired activities of daily living were independent of negative emotional states. Model 5 included baseline health behaviours. We present results as odds ratios (with 95\% confidence intervals [CIs]) for the tertiles with medium and low enjoyment of life, using the tertile with high enjoyment of life as the reference group. We repeated analyses with exclusion of participants who were aged 70 years or older at baseline, because previous work has focused on older groups ${ }^{6.16}$ We carried out a sensitivity analysis after excluding participants who had a single impaired activity of daily living at baseline, thus assessing the risk of transition from 0 to 2 or more impaired activities of daily living.

We analyzed the association between enjoyment of life at baseline and changes in gait speed over 8 years with analysis of covariance. Model 1 included baseline gait speed as a covariate, and subsequent models added demographic factors, baseline health indicators, depression and health behaviour as in the analyses of impaired activities of daily living. Additionally, we controlled for impaired activities of daily living and impaired 
mobility at baseline, to establish whether associations with enjoyment of life were independent of disability. We repeated the analysis after exclusion of participants aged 70 years or older at baseline. Results are presented as adjusted mean gait speeds with standard error of the mean. Finally, we used binary logistic regression to test whether participants who did the timed walk at baseline but not on follow-up $(n=234)$ differed in enjoyment of life from the remainder.

\section{Results}

The characteristics of the full study sample are shown in Table 1. Enjoyment of life was significantly higher among participants aged 60-69

\begin{tabular}{|c|c|c|c|c|}
\hline \multirow[b]{2}{*}{ Characteristic } & \multicolumn{3}{|c|}{ Baseline enjoyment of life, no. (\%)* } & \multirow[b]{2}{*}{$p$ value } \\
\hline & $\begin{array}{c}\text { Low } \\
n=738\end{array}$ & $\begin{array}{l}\text { Medium } \\
n=1792\end{array}$ & $\begin{array}{c}\text { High } \\
n=669\end{array}$ & \\
\hline Sex & & & & 0.7 \\
\hline Male & $314(42.5)$ & $775(43.2)$ & $292(43.6)$ & \\
\hline Female & $424(57.5)$ & $1017(56.8)$ & 377 (56.4) & \\
\hline Age, yr & & & & 0.001 \\
\hline $60-69$ & $424(57.5)$ & 1009 (56.3) & $468(70.0)$ & \\
\hline $70-79$ & $254(34.4)$ & $621(34.7)$ & $175(26.2)$ & \\
\hline$\geq 80$ & $60(8.1)$ & $162(9.0)$ & $26 \quad(3.9)$ & \\
\hline Ethnicity, nonwhite & $15(2.0)$ & $17(0.9)$ & $5 \quad(0.7)$ & 0.02 \\
\hline Education & & & & $<0.001$ \\
\hline Lower & $386(52.3)$ & $746(41.6)$ & $201(30.0)$ & \\
\hline Intermediate & $235(31.8)$ & $651(36.3)$ & $243(36.3)$ & \\
\hline Higher & $117(15.9)$ & $395(22.0)$ & $225(33.6)$ & \\
\hline Wealth quintile & $n=715$ & $n=1757$ & $n=662$ & $<0.001$ \\
\hline 1 (lowest) & $146(20.4)$ & $212(12.1)$ & $57(8.6)$ & \\
\hline 2 & $154(21.5)$ & $298(17.0)$ & $99(15.0)$ & \\
\hline 3 & $159(22.2)$ & $380(21.6)$ & $129(19.5)$ & \\
\hline 4 & $143(20.0)$ & $417(23.7)$ & $139(21.0)$ & \\
\hline 5 (highest) & $113(15.8)$ & $450(25.6)$ & $238(36.0)$ & \\
\hline Marital status, married & $419(56.8)$ & $1192(66.5)$ & $519(77.6)$ & $<0.001$ \\
\hline Paid employment & $83(11.2)$ & $229(12.8)$ & $119(17.8)$ & $<0.001$ \\
\hline Limiting, longstanding illness & $391(53.0)$ & $563(31.4)$ & $65 \quad(9.7)$ & $<0.001$ \\
\hline Coronary heart disease & $140(19.0)$ & $246(13.7)$ & 42 (6.3) & $<0.001$ \\
\hline Cancer & $49(6.6)$ & $118(6.6)$ & $42(6.3)$ & 0.8 \\
\hline Diabetes & $81(11.0)$ & $108(6.0)$ & $25 \quad(3.7)$ & $<0.001$ \\
\hline Arthritis & $326(44.2)$ & $579(32.3)$ & $147(22.0)$ & $<0.001$ \\
\hline Stroke & $52(7.0)$ & $70 \quad(3.9)$ & $8(1.2)$ & $<0.001$ \\
\hline Chronic lung disease & $72(9.8)$ & $108(6.0)$ & $17 \quad(2.5)$ & $<0.001$ \\
\hline History of depressive illness & $50(6.8)$ & $49(2.7)$ & $16(2.4)$ & $<0.001$ \\
\hline Elevated depressive symptoms & $317(43.0)$ & $299(16.7)$ & $28(4.2)$ & $<0.001$ \\
\hline Current smoker & $131(17.8)$ & $199(11.1)$ & $59(8.8)$ & $<0.001$ \\
\hline Vigorous or moderate activity $>1 / \mathrm{wk}$ & $385(52.2)$ & $1150(64.2)$ & $520(77.7)$ & $<0.001$ \\
\hline Alcohol consumption daily or almost daily & $183(24.8)$ & $519(29.0)$ & $228(34.1)$ & $<0.001$ \\
\hline Impaired mobility & $570(77.2)$ & $1094(61.0)$ & $246(36.8)$ & $<0.001$ \\
\hline$\geq 2$ impaired activities of daily living & $147(19.9)$ & $123(6.9)$ & $9(1.3)$ & $<0.001$ \\
\hline Gait speed, mean $\pm S D, \mathrm{~m} / \mathrm{s}$ & $0.79 \pm 0.29$ & $0.90 \pm 0.27$ & $1.01 \pm 0.26$ & $<0.001$ \\
\hline
\end{tabular}


years, those with greater education and wealth, and those who were married and in paid employment. Lower levels of enjoyment were more common among participants with limiting, longstanding illness; coronary heart disease; diabetes; arthritis; stroke; chronic lung disease; a history of depression; and elevated depressive symptoms at baseline. Smoking, physical inactivity and less than daily alcohol consumption were also associated with lower enjoyment of life. The results for the samples included in the analyses of impaired activities of daily living and gait speed are described in Appendices 2 and 3, available at www.cmaj.ca/lookup/suppl /doi:10.1503/cmaj.131155/-/DC1. The differences between enjoyment-of-life groups observed in the full sample were replicated in these analytic samples.

\section{The development of impaired activities of daily living}

At baseline, there was a strong association between impaired activities of daily living and enjoyment of life; $19.9 \%$ of participants in the tertile with low enjoyment of life had 2 or more impaired activities of daily living, compared with $6.9 \%$ in the medium and $1.3 \%$ in the high enjoyment-of-life tertiles. Over the 8-year follow-up period, $323(11.1 \%)$ participants with 0-1 impaired activities of daily living at baseline developed 2 or more impaired activities of daily living. These participants reported an average of 3 impaired activities of daily living (range 2-6). The incidence of new impaired activities of daily living was $4.4 \%, 11.7 \%$ and $16.8 \%$ in the tertiles with high, medium and low enjoyment of life, respectively. The odds of impaired activities of daily living developing were 3.17 (95\% CI 2.034.95) for the low compared with high enjoymentof-life tertile, adjusted for age, sex and having a single impaired activity of daily living at baseline (Figure 1). Adjustment for demographic factors and baseline health reduced the odds to 2.10. Adjustment for depressive symptoms had little impact. In the final model, the odds of developing 2 or more impaired activities of daily living remained significant for individuals in the medium $(1.73,95 \%$ CI 1.13-2.64) and low (1.83, 95\% CI 1.13-2.96) enjoyment-of-life tertiles. Other independent predictors of future impaired activities of daily living in the final model were greater age; less wealth; having limiting, longstanding illness; baseline diabetes, arthritis and stroke; having a single impaired activity of daily living at baseline; and physical inactivity.

We repeated the analyses for participants younger than 70 years at baseline (Appendix 4, available at www.cmaj.ca/lookup/suppl/doi:10 .1503/cmaj.131155/-/DC1), and the associations with positive well-being were more striking.

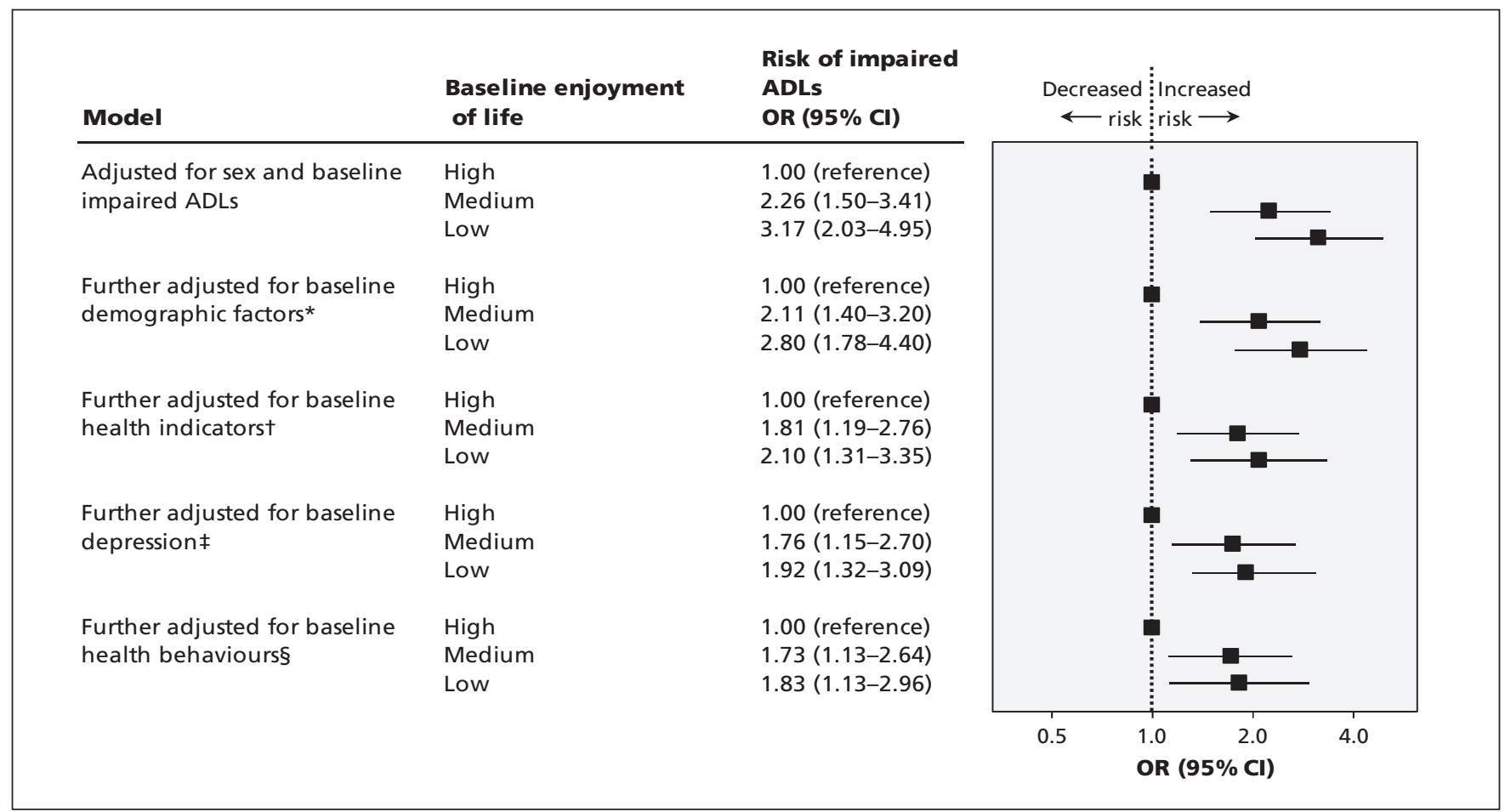

Figure 1: Baseline enjoyment of life and risk of impaired activities of daily living (ADLs) 8 years later. $\mathrm{Cl}=$ confidence interval, OR = odds ratio. *Demographic factors include wealth, education, ethnicity, marital status and employment. tHealth indicators include limiting, longstanding illness; coronary heart disease; cancer; stroke; chronic lung disease; diabetes; and arthritis. $¥ D e p r e s s i o n$ includes depressive illness and current symptoms of depression. §Health behaviours include physical activity, smoking and alcohol intake. 
Thus, in the fully adjusted model, the odds of 2 or more impaired activities of daily living were 3.53 (95\% CI 1.56-7.95) for the low enjoyment-of-life tertile, compared with 1.83 in the full sample. The sensitivity analysis excluding participants at baseline with 1 impaired activity of daily living showed comparable results to those in the full dataset (Appendix 5, available at www.cmaj.ca /lookup/suppl/doi:10.1503/cmaj.131155/-/DC1).

\section{Changes in gait speed over 8 years}

Gait speed was 0.92 (SD 0.28) m/s at baseline and declined by $12 \%$ to 0.81 (SD 0.27 ) $\mathrm{m} / \mathrm{s}$ 8 years later. Participants who did not complete the walk test at baseline had more limiting illness, diabetes, arthritis and mobility impairment, and reported less physical activity than the remainder. Baseline gait speed was associated with enjoyment of life, with means adjusted for all covariates of $0.94,0.90$ and $0.86 \mathrm{~m} / \mathrm{s}$ for the high, medium and low enjoyment-of-life groups $(p<0.001)$. Baseline enjoyment of life also predicted gait speed 8 years later (Figure 2). The mean difference adjusted for baseline gait speed between high and low enjoyment-of-life tertiles was $0.10 \mathrm{~m} / \mathrm{s}$. This difference fell to $0.05 \mathrm{~m} / \mathrm{s}$ in the full model that included demographic factors, health at baseline, depression, impaired mobility and activities of daily living, and health behaviours. Nevertheless, about $50 \%$ of the difference between high and low enjoyment-of-life tertiles remained unexplained. Other independent predictors in the final model were baseline gait speed, age, sex, wealth, employment status, arthritis and diabetes at baseline, baseline mobility impairment, physical activity and alcohol consumption. The same pattern of results emerged from the analysis of participants younger than 70 years (Appendix 6, available at www.cmaj.ca/lookup /suppl/doi:10.1503/cmaj.131155/-/DC1).

The gait speed of 2965 participants was measured at baseline, and 2507 of these participants completed the walk test 8 years later. Those who completed the measure only at baseline were more likely to be from the low compared with high enjoyment-of-life tertile (adjusted odds

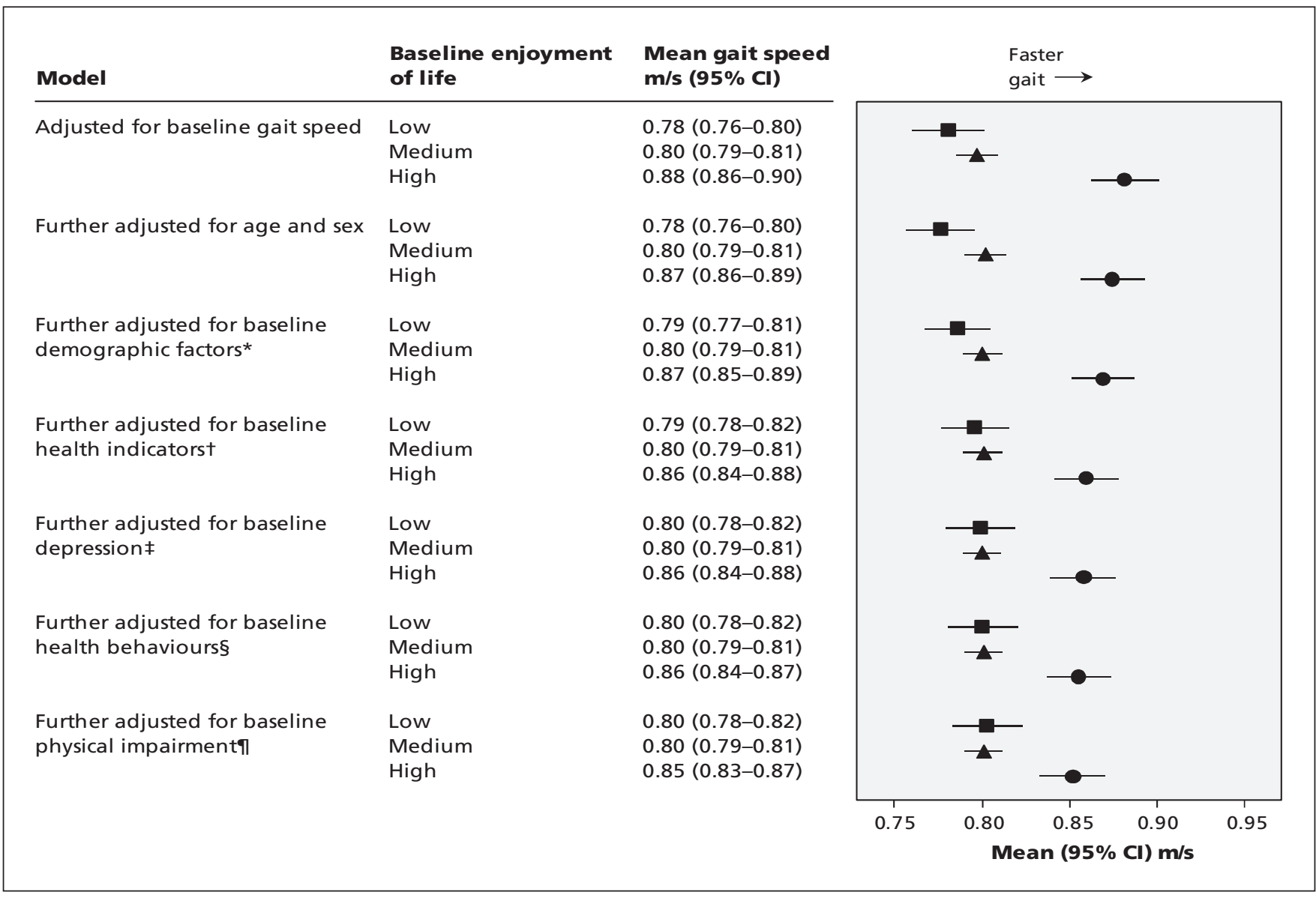

Figure 2: Baseline enjoyment of life and gait speed 8 years later among groups with low (square), medium (triangle) and high (circle) enjoyment of life. $\mathrm{Cl}=$ confidence interval. *Demographic factors include wealth, education, ethnicity, marital status and employment. tHealth indicators include limiting, longstanding illness; coronary heart disease; cancer; stroke; chronic lung disease; diabetes; and arthritis. ‡Depression includes depressive illness and current symptoms of depression. §Health behaviours include physical activity, smoking and alcohol intake. ПPhysical impairment includes impaired mobility and activities of daily living. 
ratio 1.76 , 95\% CI $1.20-2.58)$. Of the respondents, $23.6 \%$ in the low, $14.9 \%$ in the medium and $8.2 \%$ in the high enjoyment-of-life tertiles did not complete the timed walk on follow-up.

\section{Interpretation}

We tested the association between enjoyment of life and physical function 8 years later using both self-reported and objective indicators of function. Greater enjoyment of life was associated with reduced risk of developing impaired activities of daily living and with a slower decline in gait speed. Associations were partly accounted for by baseline differences in sociodemographic profile, health status and health behaviours; however, a substantial proportion of the variance in risk remained unexplained. The protective effect of enjoyment of life is not simply the reverse of psychological distress, because the covariates included measures of clinical depression and symptoms of depression. Associations are not limited to older age, because similar findings emerged for those in the younger age spectrum (60-69 yr). We also checked whether the association with gait speed was the result of selective completion of the timed walk at follow-up. However, our sensitivity analysis indicated that noncompletion of the follow-up walk test was more common among participants with low rather than high enjoyment of life. The main results were not, therefore, due to participants with high enjoyment of life not completing the follow-up test.

There is growing interest in subjective wellbeing as an indicator of societal flourishing. ${ }^{17}$ Measures of positive affective well-being derived from depression questionnaires have previously been associated with activities of daily living and walking speed changes over 2 years, ${ }^{6-8}$ but our measure of enjoyment of life was distinct from items on depression inventories, which allowed us to control for depressive symptoms. The more extended follow-up period in these analyses reduces the likelihood of reverse causality. Steptoe and Wardle ${ }^{12}$ have previously shown that enjoyment of life predicted survival over a 7year period, independently of covariates. The present results indicate that future functional decline is associated with enjoyment of life. The finding that comparable results emerged for selfreported activities of daily living and gait speed indicates that the association with enjoyment of life cannot be attributed to reporting biases, but reflects objective differences in functional capacity. An independent analysis of the English Longitudinal Study of Ageing has shown that enjoyment of life also predicts incident frailty over a 4-year period. ${ }^{18}$

\section{Limitations}

This was an observational study, so causal conclusions cannot be drawn. The associations we found may have been due to other unmeasured variables or to common processes underlying both enjoyment of life and decline in function. The 20 covariates in these analyses cover the main sources of confounding, but more precise assessments of baseline function, adiposity and health would have been desirable. We didn't assess comorbidities directly, but rather used patient reports of doctor diagnoses; however, these reports have validity in large-scale population studies. ${ }^{19,20}$ Cognitive impairment was not included as a covariate, although it is a known predictor of functional decline. We did not evaluate the clinical significance of the findings. Objective measures of function were limited to walking speed, and biomarkers were not collected. A small proportion of participants did not carry out the walk test at baseline because of health conditions or disabilities. However, because gait speed was positively related to enjoyment of life at baseline, exclusion of these participants from the analysis is unlikely to have biased the results in favour of our hypotheses.

\section{Conclusion}

Slow walking speed is considered an early marker of disability and frailty, ${ }^{21}$ as well as a predictor of dementia, ${ }^{22}$ admission to a long-term health facility and death. ${ }^{9}$ We did not evaluate the clinical significance of the findings. However, an $80 \%$ increase in odds of acquiring 2 or more impairments in activities of daily living over 8 years among people with low compared with high enjoyment of life is striking, and the differences in walking speed are important for everyday function. Our finding that associations remain significant after covariates are taken into account suggests that direct biological correlates of enjoyment of life may be involved. ${ }^{23,24}$ Our results provide further evidence that enjoyment of life is relevant to the future disability and mobility of older people. Efforts to enhance wellbeing at older ages may have benefits to society and health care systems. ${ }^{25}$

\section{References}

1. Steptoe A, Wardle J. Positive affect measured using ecological momentary assessment and survival in older men and women. Proc Natl Acad Sci U S A 2011;108:18244-8.

2. Diener E, Chan MY. Happy people live longer: subjective wellbeing contributes to health and longevity. Applied Psychol Health Well Being 2011;3:1-43.

3. Ostir GV, Markides KS, Peek MK, et al. The association between emotional well-being and the incidence of stroke in older adults. Psychosom Med 2001;63:210-5.

4. Boehm JK, Kubzansky LD. The heart's content: the association between positive psychological well-being and cardiovascular health. Psychol Bull 2012;138:655-91.

5. Steptoe A, Dockray S, Wardle J. Positive affect and psychobiological processes relevant to health. J Pers 2009;77:1747-76. 
6. Hirosaki M, Ishimoto Y, Kasahara Y, et al. Positive affect as a predictor of lower risk of functional decline in communitydwelling elderly in Japan. Geriatr Gerontol Int 2013;13:1051-8.

7. Ostir GV, Markides KS, Black SA, et al. Emotional well-being predicts subsequent functional independence and survival. $J$ Am Geriatr Soc 2000;48:473-8.

8. Park-Lee E, Fredman L, Hochberg M, et al. Positive affect and incidence of frailty in elderly women caregivers and noncaregivers: results of Caregiver-Study of Osteoporotic Fractures. J Am Geriatr Soc 2009;57:627-33.

9. Studenski S, Perera S, Patel K, et al. Gait speed and survival in older adults. JAMA 2011;305:50-8.

10. Steptoe A, Breeze E, Banks J, et al. Cohort profile: The English Longitudinal Study of Ageing. Int J Epidemiol 2013;42: 1640-8.

11. Hyde M, Wiggins RD, Higgs P, et al. A measure of quality of life in early old age: the theory, development and properties of a needs satisfaction model (CASP-19). Aging Ment Health 2003;7:186-94.

12. Steptoe A, Wardle J. Enjoying life and living longer. Arch Intern Med 2012;172:273-5.

13. Wallace RB, Herzog AR. Overview of the health measures in the Health and Retirement Study. J Hum Resour 1995;30(supplement):S84-107.

14. Steffick DE. Documentation of affective functioning measures in the Health and Retirement Study. Ann Arbor (MI): Survey Research Center University of Michigan; 2000.

15. Demakakos P, Pierce MB, Hardy R. Depressive symptoms and risk of type 2 diabetes in a national sample of middle-aged and older adults: the English Longitudinal Study of Ageing. Diabetes Care 2010;33:792-7.

16. Pitkala KH, Laakkonen ML, Strandberg TE, et al. Positive life orientation as a predictor of 10-year outcome in an aged population. J Clin Epidemiol 2004;57:409-14.

17. OECD guidelines on measuring subjective well-being. Paris (France): Organisation for Economic Co-operation and Development; 2013. Available: http://dx.doi.org/10.1787/9789264191655 -en (accessed 2013 July 24).

18. Gale CR, Cooper C, Deary IJ, et al. Psychological well-being and incident frailty in men and women: the English Longitudinal Study of Ageing. Psychol Med 2013;44:697-706.

19. Baumeister H, Kriston L, Bengel J, et al. High agreement of self-report and physician-diagnosed somatic conditions yields limited bias in examining mental-physical comorbidity. J Clin Epidemiol 2010;63:558-65.

20. Kriegsman DM, Penninx BW, van Eijk JT, et al. Self-reports and general practitioner information on the presence of chronic dis- eases in community dwelling elderly. a study on the accuracy of patients' self-reports and on determinants of inaccuracy. J Clin Epidemiol 1996;49:1407-17.

21. Guralnik JM, Ferrucci L, Pieper CF, et al. Lower extremity function and subsequent disability: consistency across studies, predictive models, and value of gait speed alone compared with the short physical performance battery. J Gerontol A Biol Sci Med Sci 2000; 55:M221-31.

22. Abellan van Kan G, Rolland Y, Gillette-Guyonnet S, et al. Gait speed, body composition, and dementia. The EPIDOS-Toulouse cohort. J Gerontol A Biol Sci Med Sci 2012;67:425-32.

23. Steptoe A, Wardle J, Marmot M. Positive affect and healthrelated neuroendocrine, cardiovascular, and inflammatory processes. Proc Natl Acad Sci U S A 2005;102:6508-12.

24. Dockray S, Steptoe A. Positive affect and psychobiological processes. Neurosci Biobehav Rev 2010;35:69-75.

25. Bolier L, Haverman M, Westerhof GJ, et al. Positive psychology interventions: a meta-analysis of randomized controlled studies. BMC Public Health 2013;13:119.

Affiliation: Department of Epidemiology and Public Health, University College London, London, UK

Contributors: Andrew Steptoe, Cesar de Oliveira and Panayotes Demakakos conceived the study. Andrew Steptoe and Paola Zaninotto carried out the statistical analysis and drafted the article. All authors made substantial contributions to the analysis and interpretation of data, revised the article and approved the final version submitted for publication. Andrew Steptoe is the guarantor.

Funding: The English Longitudinal Study of Ageing was developed by a team of researchers based at University College London, the Institute for Fiscal Studies and the National Centre for Social Research. The funding is provided by the National Institute on Aging (grant nos. 2RO1AG7644-01A1 and 2RO1AG017644) and a consortium of UK government departments coordinated by the Office for National Statistics. The funders of this research had no input into the design and conduct of the study; collection, management, analysis or interpretation of data; preparation, review or approval of the manuscript; or the decision to submit the manuscript for publication. Andrew Steptoe receives funding from the British Heart Foundation. 